\title{
REVIEW ARTICLE OPEN Centrosome dysfunction: a link between senescence and tumor immunity
}

\author{
Qi Wu (D) ${ }^{1}$, Bei $\mathrm{Li}^{1,2}$, Le $\mathrm{Liu}^{3}$, Shengrong Sun ${ }^{1}$ and Si Sun ${ }^{4}$
}

Centrosome aberrations are hallmarks of human cancers and contribute to the senescence process. Structural and numerical centrosome abnormalities trigger mitotic errors, cellular senescence, cell death, genomic instability and/or aneuploidy, resulting in human disorders such as aging and cancer and affecting immunity. Interestingly, centrosome dysfunction promotes the secretion of multiple inflammatory factors that act as pivotal drivers of senescence and tumor immune escape. In this review, we summarize the forms of centrosome dysfunction and further discuss recent advances indicating that centrosome defects contribute to acceleration of senescence progression and promotion of tumor cell immune evasion in different ways.

\section{INTRODUCTION}

In animal cells, the centrosome is the major microtubule organizing center (MTOC). Centrosomes promote the production of bipolar mitotic spindles and supply a matrix of primary cilia in various cell types. In addition to these structural functions, centrosomes and primary cilia have also evolved into essential signaling hubs. ${ }^{1}$ In normal cells, a pair of centrioles comprises the centrosome, which is embedded in the pericentriolar material (PCM), an electron-dense amorphous matrix. The PCM supplies sites for microtubule nucleation, thus determining the number and composition of microtubules during the cell cycle. Large-scale proteomic studies have revealed that there are more than 200 centrosome-associated proteins; ${ }^{2}$ however, the exact functions of most of these proteins are still unknown and require further investigation.

The structure, function and number of centrosomes are strictly controlled within cells. To form an effective bipolar mitotic spindle, the centrosome must duplicate in the $S$ phase, and further centrosome maturation occurs when additional PCM material is recruited during the $\mathrm{G} 2$ phase of the cell cycle. Finally, the two centrosomes must separate upon mitotic entry. Like DNA replication, centrosome duplication is semiconservative and occurs only once per cell cycle. A rigorous regulatory network ensures that the two processes are carried out in an ordered manner during the late $\mathrm{G} 1$ phase. $^{2}$ Therefore, centrosome number determination and centrosome duplication are associated in each cell. Proper centrosome duplication, maturation and separation are fundamental for the formation of bipolar spindles and, therefore, for faithful chromosome segregation. As a consequence, the accurate inheritance of genetic material might be hindered by centrosome dysfunction (CD). ${ }^{3}$ Furthermore, centrosome abnormalities can lead to chromosomal instability (CIN), an ongoing process of gain and/or loss of whole chromosomes. ${ }^{4,5}$ In turn, this can lead to aneuploidy, a state in which too many or too few chromosomes are present. ${ }^{6}$ Therefore, abnormal chromosome content, a biomarker of human cancers, is associated with aneuploidy and CIN. ${ }^{4,7}$

Initially, cellular senescence was identified as a mostly irreversible type of growth arrest that occurs when cleavable cells undergo extensive intrinsic and/or extrinsic damage, including oncogenic activation, mitochondrial dysfunction, radiation damage, oxidative and genotoxic stress, and chemotherapeutic agent-induced damage. ${ }^{8}$ At the cellular level, it has been determined that there is a relationship among senescence, CIN and aneuploidy in various cell types in different species. ${ }^{9}$ Interestingly, increasing evidence supports a connection between $\mathrm{CD}$ and aging, indicating that centrosomes may have direct or indirect effects on senescence. ${ }^{10}$

According to the results of Boveri's research a century ago, centrosome numerical abnormalities may result in tumorigenesis. ${ }^{11}$ Boveri created fertilized eggs harboring extra centrosomes, and these eggs appeared to undergo multipolar mitoses. In Boveri's model, centrosome abnormalities caused chromosome mis-segregation during mitosis, which triggered malignancy. Importantly, increasing amounts of circumstantial evidence have suggested that aberrant centrosome numbers and functions are associated with aneuploidy and human cancers, ${ }^{3}$ and the causal relationships between centrosome abnormalities and tumorigenesis have been characterized. ${ }^{12,13}$ Centrosome abnormalities in cell culture often result in aberrant spindle assembly and damaged microtubule-kinetochore attachments. ${ }^{14}$ As demonstrated by Jusino et al., centrosome anomalies and tumorigenesis are connected in that centrosome dysregulation leads to persistent CIN. ${ }^{15}$ Interestingly, chromosome segregation errors and replication stress resulting from CIN can generate doublestranded DNA (dsDNA) and revitalize the innate immunological

\footnotetext{
${ }^{1}$ Department of Breast and Thyroid Surgery, Renmin Hospital of Wuhan University, Wuhan, Hubei, China; ${ }^{2}$ Department of Pathology, Renmin Hospital of Wuhan University,

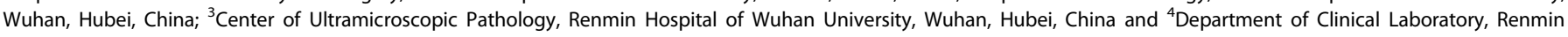
Hospital of Wuhan University, Wuhan, Hubei, China

Correspondence: Shengrong Sun (sun137@sina.com) or Si Sun (59333173@qq.com)

These authors contributed equally: Qi Wu, Bei Li
}

Received: 9 March 2020 Accepted: 8 June 2020

Published online: 30 June 2020 
response by activating cyclic GMP-AMP synthase (cGAS)-stimulator of interferon genes (STING) signaling. ${ }^{16,17}$ This recent discovery not only enhances our understanding of the effects of $C D$ on tumor development but also elucidates the consequences of $C D$ associated with the interplay between cancer cells and the immune microenvironment. Here, we explore the impacts of such recent advances on our current understanding of the causes and consequences of centrosome abnormalities with regard to senescence and tumor immunity.

\section{MECHANISMS OF CENTROSOME ABNORMALITIES}

Increasing amounts of evidence have shown that centrosome abnormalities in cells are fairly universal among solid tumors and in many hematopoietic malignancies. For example, approximately $80 \%$ of invasive breast tumors exhibit amplified centrosomes. ${ }^{18}$ Consistent with these findings, centrosome abnormalities have been detected in $72 \%$ of patients with B-acute lymphoblastic leukemia. ${ }^{19}$ Importantly, centrosome amplification is strongly associated with the development of aneuploidy and CIN in a variety of tumor types. ${ }^{18-21}$ Centrosome abnormalities in tumors can be roughly divided into numerical abnormalities and structural abnormalities (Fig. 1). Increases in centrosome copy numbers cause numerical abnormalities; such increases can be caused by mistakes in centrosome replication or by failure of cell division. Centrosome numbers have been found to be significantly associated with tumor grade and the proliferative index in myeloid leukemia, non-Hodgkin's lymphoma and multiple myeloma. ${ }^{22-24}$ Although the characteristics of structural centrosome abnormalities have rarely been addressed, the most common defect is an increase in centrosome size due to PCM expansion. ${ }^{25}$ In malignant breast cancer, structural centrosome abnormalities involve mainly overly long centrioles that enhance centrosome amplification through both centriole fragmentation and ectopic procentriole formation. ${ }^{26}$ In addition, structural centrosome aberrations induced by ninein-like protein (NLP) can induce reorganization of the cytoskeleton and increase cell stiffness to trigger cell dissemination. ${ }^{27}$ Although structural and numerical abnormalities often coexist in tumors and induce some of the same effects, they can also facilitate different cell mechanistic behaviors, as discussed below. 27,28

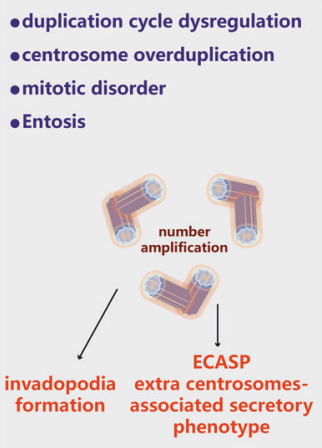

- the changed amount of centrosome components abnormal locations of core - abnorma lc error junction among core proteins

Fig. 1 Mechanisms of centrosome aberrations in cancer. The mechanisms of centrosome number abnormalities include duplication cycle dysfunction, centrosome overduplication, mitotic disorder and entosis. These processes result in the formation of invadopodia and establishment of an extra centrosome-associated secretory phenotype (left). The mechanisms of structural centrosome abnormalities include changes in the amounts of centrosome components, abnormal localization of core proteins, and aberrant binding among core proteins; these changes lead to cell extrusion and dissemination of mitotic cells (right)

\section{NUMERICAL CENTROSOME ABNORMALITIES}

Numerical abnormalities, including centrosome amplification, are the most common centrosome defects in tumors. Centrosome amplification can be induced through many mechanisms, such as cytokinesis failure, mitotic slippage, cell-cell fusion, centriole overduplication and de novo centriole assembly. ${ }^{29}$ Various potential mechanisms can explain the variety of proteins, such as tumor suppressors and oncogenic proteins, involved in centrosome amplification in the context of cancer. ${ }^{30}$ Importantly, centriole overduplication, rather than cytokinesis failure or cell-cell fusion, is considered the predominant contributor to centrosome amplification, as indicated by the results of an analysis of centrosomes in primary human melanomas. In that study, CEP170 staining was used to assess whether centrosomes contained mature centrioles. Overduplicated centrioles were expected to not harbor CEP170, while extra centrioles resulting from cell doubling events were expected to harbor CEP170. The authors found a lower percentage of CEP170-positive centrosomes in melanomas than in benign samples. ${ }^{31}$

Dysregulation of the centrosome duplication cycle is one of the crucial pathways leading to centrosome amplification. The duplication cycle is partially, but strictly, regulated by many pivotal regulators; in normal cells, centrosome amplification is prevented by numerous factors that regulate centrosome duplication positively or negatively. ${ }^{32}$ However, this strictly controlled centrosome cycle depends on only a few evolutionarily conserved core proteins. ${ }^{33}$ For example, polo-like kinase (PIk) 4 is an important core protein that is a major regulator of centrosome duplication. ${ }^{34}$ The activity of Plk4 is a key factor in the regulation of centriole number, as indicated by the findings that excessive activity of Plk4 results in additional centrioles, ${ }^{35}$ while Plk4 depletion decreases centriole numbers. ${ }^{35,36}$ To guarantee correct centriole duplication, Plk4 levels are primarily regulated by SCFBTrCP/ubiquitin-dependent proteolysis, ${ }^{37}$ which is partially controlled by autophosphorylation. ${ }^{38,39}$ P53 negatively regulates Plk4 mRNA levels by recruiting histone deacetylase (HDAC) repressors to the promoter of Plk4. ${ }^{40}$ Therefore, loss of p53 may promote centrosome amplification by increasing the levels of Plk4. This mechanism is consistent with the observation that deletion of p53 is related to increased centrosome numbers in mouse fibroblasts. ${ }^{41}$ However, a recent study has demonstrated that the numbers of centrosomes in the brains of $\mathrm{p} 53^{-/-}$mice are normal, ${ }^{42}$ showing that deletion of p53 is insufficient to cause centrosome amplification. Therefore, p53 may affect amplification differently in different tissues, suggesting that other mechanisms may be more influential than p53 signaling with regard to centrosome amplification. ${ }^{43}$

Tetraploidy, which can be caused by abnormal centrosome replication or by failure of cell division, was first discovered in fertilized eggs by Theodor Boveri. ${ }^{11}$ Notably, previous studies have revealed that centrosome amplification occurs in cells with telomere-driven tetraploidy, which can result from cytokinesis failure, mitotic slippage, endoreduplication or cell-cell fusion and can promote transformation. ${ }^{44,45}$ Although these studies did not evaluate the effects of centrosome amplification, cells originating from $\mathrm{p} 53^{-/-}$tetraploid tumors have been found to have increased numbers of centrosomes. ${ }^{46}$ In one study, tetraploid cells isolated from $\mathrm{p} 53^{-1-}$ mouse mammary epithelial cells (MMECs) undergoing transient cytokinesis failure caused mammary epithelial tumorigenesis upon subcutaneously implantation into nude mice. ${ }^{46}$ However, some findings of a recent in vitro study contradict the supposition that tetraploid tumors exhibit centrosome overduplication: specifically, tetraploidized $\mathrm{p} 53^{-/-} \mathrm{HCT} 116$ cells fail to cleave and cease to proliferate, resulting in long-term loss of centrosomes in cultured cells. ${ }^{47}$ One possible reason for this result is that transient blockade of cytokinesis may generate aneuploid cells and subsequently lead to propagation of both diploid and tetraploid $\mathrm{p} 53^{-/-}$cells. On the other hand, the oncogene Mos plays 


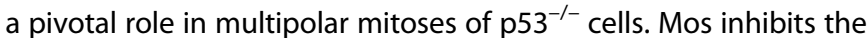
coalescence of supernumerary centrosomes to induce frequent tetraploidization, and knockdown of Mos stagnates multipolar mitoses and drives $\mathrm{CIN}$ in $\mathrm{p} 53^{-/-}$cells. ${ }^{48}$ Taken together, these findings indicate that there is not a simple correspondence between the production of additional centrosomes and the continued maintenance of extra centrosomes. Clearly, centrosome amplification itself is detrimental. Previous studies have shown that additional centrosomes disappear spontaneously in newly originated tetraploid cells during continuous passage in culture. ${ }^{49}$ In addition, other permissive conditions must coexist, such as conditions related to specific cell types and genetic changes, for cytokinesis failure to induce tetraploidy in cells and thus to cause long-term stable centrosome amplification.

Notably, entosis is observed in cells found in the urine and ascites fluid of cancer patients ${ }^{50}$ and is identified by the presence of cell-in-cell structures. ${ }^{51}$ Entosis occurs mainly in epithelial tumor cells under specific conditions, such as abnormal proliferation, glucose exhaustion, ${ }^{52}$ matrix detachment, ${ }^{51}$ and mitotic stress. ${ }^{53}$ The effects of entosis on tumorigenesis remain unclear; however, entosis may induce aneuploidy as cells are engulfed ${ }^{54}$ and may supply nutrition for tumor growth. ${ }^{52}$ Cell division, detachment or death are the likely ends for engulfed cells. However, deficiencies in autophagosome-lysosome function and apoptosis can cause internalized cells to be released by their host cells and reappear unharmed. ${ }^{51,55}$ In human breast tumors, live cells are internalized by entosis, but the centrosomes of native cells are preserved in the host cells. Furthermore, the preserved centrosomes continue to function during the cell cycle of the host cells, thereby disrupting the formation of the contractile ring during host cell division. Consequently, cytokinesis failure occurs frequently, producing binucleate cells that induce increases in centrosome number. ${ }^{54}$ These observations suggest the existence of a novel relationship between cytokinesis failure and centrosome abnormalities.

\section{STRUCTURAL CENTROSOME ABNORMALITIES}

Centrosome structural defects can be roughly classified into two groups: defects in centriole structure and defects in the amounts of PCM components (Fig. 1). Alterations in centriole size are the most straightforward structural defects and are usually observed as increases in centriole length. How centriole structural defects arise in cancer cells remains unclear; however, a recent study has demonstrated that centrosome amplification can be triggered by severe centriole overelongation, which results in the formation of overactive centrosomes that nucleate more microtubules than normal centrosomes. ${ }^{26}$ Alterations in the expression levels of genes that control centriole structure may underlie structural defects. For instance, upregulation or downregulation of the expression of centrosomal components may result in abnormalities in centriole structure; in a few model systems, overexpression of CPAP/SAS-4 has been found to increase centriole length, which influences normal centromeric assembly. ${ }^{56,57}$

It is not easy to identify specific structural defects of centrioles in cancer cells. First, given that centrioles are $0.2-0.5 \mu \mathrm{m}$ long, close to the optical resolution limits of optical microscopes, measurement of centriole length requires specific fluorescence techniques or electron microscopy. Second, it is difficult to categorize tumors on the basis of their structural defects. For instance, the amount of PCM could be deduced from the volume/ diameter of a centrosome using a pericentriolar marker, ${ }^{58}$ and an increased amount of PCM has been regarded as a structural defect. $^{59}$ However, there are two explanations for increased amounts of PCM in cells. One the one hand, an increase in PCM could be a valid interpretation of the measured data and support the classification of excess PCM as a centrosome structural defect. $^{58}$ On the other hand, the observation could be a result of additional centrosome clustering during interphase, which is considered a numerical defect. ${ }^{58,60}$ These two possibilities can be assessed only by authentic centriole labeling. Incorrect and opposite categorization can also occur because it is thought that increased centriole length results in centriole fragmentation in vitro. ${ }^{57}$ Therefore, determining the origins of centrosome structural changes via only fixed-cell imaging is not an easy task and is further complicated by the fact that most tumor studies consider only PCM markers without considering centriole markers. Most importantly, it is necessary to systematically identify and classify centrosome abnormalities through specific methods in order to better describe centrosome abnormalities in human tumors and elucidate how these centrosomal defects arise.

\section{CENTROSOME ABERRATIONS AND SENESCENCE}

Senescence, or aging, is a natural biological process that occurs in all living organisms. The characteristics of aging include disruptions to cellular metabolism and function that change with time to ultimately cause permanent cell cycle arrest and cell death. Although senescence is observed both in whole organisms ${ }^{61,62}$ and in individual cells, ${ }^{63}$ the molecular and cellular mechanisms of senescence are still unclear. At the cellular level, it has been determined that senescence is correlated with $\mathrm{CIN}$ and aneuploidy in different cell types in different species. ${ }^{9,64}$ Some physiological stresses like oxidative stress are considered to play roles in the aging process, ${ }^{65}$ DNA damage, ${ }^{66}$ telomere shortening, ${ }^{67}$ high levels of tumor suppressor gene expression ${ }^{68}$ and oncogenic activation. ${ }^{69}$ Interestingly, increasing evidence has revealed a relationship between $C D$ and senescence (Fig. 2), suggesting that the centrosome could play a role in aging directly or indirectly. For example, aged porcine oocytes exhibit loss of $y$-tubulin and NuMA, which are critical PCM components of the meiotic spindle; this loss results in increased spindle abnormality and disorganization. $^{70}$ In the same way, the spindles of aged human oocytes gradually lose microtubules, which strongly suggests that centrosome structure and function are impaired during aging. ${ }^{71}$ In addition, the integrity of centrosomes and microtubules is lost in aged human oocytes ${ }^{72}$ and in aged Drosophila cells. ${ }^{73}$ Moreover, because of telomere shortening and oxidative stress, human primary fibroblasts no longer divide and instead enter replicative senescence after a limited number of cell divisions. ${ }^{63}$ These cells become senescent due to increases in the frequency of abnormal mitosis and the incidence of supernumerary centrosomes. ${ }^{9}$ Importantly, disruption of core PCM components in earlypassage mouse embryonic fibroblasts (MEFs) can induce centrosome fragmentation and initiate premature senescence, ${ }^{74}$ indicating that $C D$ alone is enough to promote the progression of cellular senescence. Another study has revealed that pericentrin and PCM1 play roles in cell cycle regulation. ${ }^{75} \mathrm{PCM}-1$ can recruit pericentrin to PCM, and inhibition of pericentrin or PCM-1 induces permanent exit from the cell cycle that is accompanied by increased expression of cellular $\beta$-galactosidase, a hallmark of cellular senescence. Similarly, depletion of other PCM components, such as Cep192 (which recruits NEDD1 to the PCM) and NEDD1 (which recruits $\gamma$-tubulin to the $P C M$ ), leads to centrosome fragmentation and premature entry into senescence. ${ }^{73}$ In summary, these findings show the interesting possibility that centrosome aberrations are types of cellular stresses that can prime cells to exit the cell cycle permanently. Future high-resolution and electron microscopy studies are necessary to identify these structural aberrations and determine their contributions to senescence.

The underlying molecular mechanism of $C D$ and its effects on cellular senescence induced by various pathological stresses have recently been explored. As shown in Fig. 2, oxidative stress induces replicative senescence of primary MEFs with age; ${ }^{65}$ however, instead of increasing in number, the centrosomes are fragmented into smaller pieces as late-passage cells begin to 


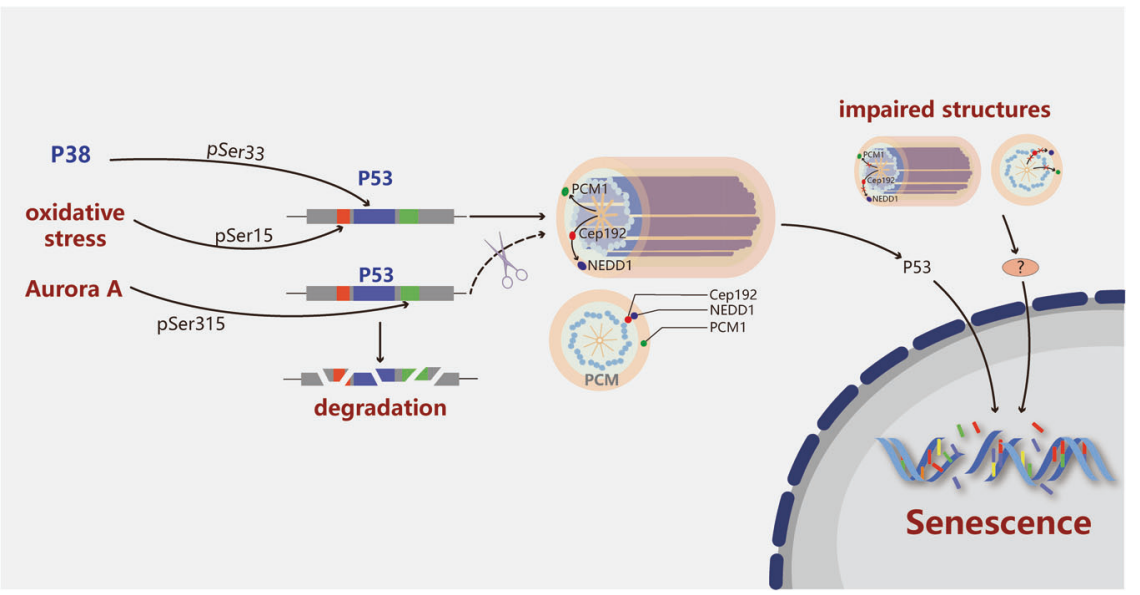

Fig. 2 Centrosome aberration-associated molecular pathways in cellular senescence. Oxidative stress and several stress-associated regulators activate p53 via phosphorylation (forming phosphorylated p53, P-p53) to stimulate the translocation of p53 to centrosomes or promote its degradation. Phosphorylation of centrosomal p53 subsequently triggers the activation of proteins that modulate the onset of senescence. In addition, dissociation of pericentrin or PCM-1 induces permanent exit from the cell cycle that is accompanied by an increase in the expression of cellular $\beta$-galactosidase, a hallmark of cellular senescence. Similarly, disruption of other PCM components, such as Cep192 (which recruits NEDD1 to the PCM) and NEDD1 (which recruits $\gamma$-tubulin to the PCM), leads to centrosome fragmentation and premature entry into the senescence pathway. The scissors indicate that p53 cannot translocate into centrosomes

undergo senescence. $^{74}$ Moreover, centrosome amplification induces an early oxidative stress response by increasing NOXmediated generation of reactive oxygen species (ROS), and high ROS levels induce a senescence-like phenotype in cells with additional centrosomes. ${ }^{28}$ P38 is a protein involved in the cellular stress response and senescence; intriguingly, p38 is activated to phosphorylate p53 at Ser33, which causes p53 to accumulate at centrosomes before it is translocated to the nucleus. ${ }^{76}$ Similar to the findings of previous studies, this finding indicates that cell cycle arrest also depends on p38 and p53 and that the arrest may be due to increased protein levels of p53 and p21 and decreased levels of phosphorylated retinoblastoma (Rb). Moreover, Aurora A and its downstream target, TACC3, localize to the PCM during mitosis. K162R mutation inactivates Aurora A, and inhibition of either Aurora A or TACC3 results in premature senescence of $p 53$ proficient tumor cells by increasing p53, p21 and hypophosphorylated Rb levels. ${ }^{77}$ The increased p53 levels may be partially explained by findings that Aurora A typically phosphorylates p53 at Ser315 to make it sensitive to degradation and that p53 becomes stable in the absence of Aurora A. ${ }^{78}$ Primary human fibroblasts undergoing replicative senescence or premature senescence induced by oxidative stress also accumulate p53 in the centrosome and exhibit simultaneous p53 phosphorylation at Ser15. ${ }^{79}$ Phosphorylation of p53 at Ser15 is critical not only for p53 localization to the centrosome but also for the default pathway of early mitosis that ensures proper cell division through ataxia telangiectasia mutated (ATM) at the centrosome. ${ }^{80}$ When the mitotic spindle performs normal function, Ser15 is rapidly dephosphorylated, and p53 remains isolated and inactive at the centrosome. However, when the spindle is impaired, p53 remains phosphorylated at Ser15; eventually, the phosphoprotein is translocated to the nucleus to trigger cell cycle arrest and cell senescence. Thus, it is possible that the phosphorylation and accumulation of $\mathrm{p} 53$ at the centrosome is a pivotal event that occurs early in the process of senescence in response to centrosome damage and other stresses. It has long been known that p53 is located on centrosomes; however, the function of p53 on these organelles is poorly understood. ${ }^{79-81}$ It will be interesting to determine the functional significance of centrosomal p53 and its differential phosphorylation states with respect to various kinases in the future. Elucidating these molecular characteristics will aid in understanding of how p53 integrates signals from different types of stresses to facilitate cellular senescence.

\section{CENTROSOME AMPLIFICATION AND TUMOR IMMUNITY}

Increasing attention is being paid to the application of effective immunotherapies for the clinical treatment of tumor patients. Molecular identification of tumor antigens is the basis of contemporary tumor immunology and cancer immunotherapy. ${ }^{82}$ A few studies have focused on the impacts of $C D$ on antitumor immune responses and the underlying molecular mechanisms (Fig. 3). Importantly, centrosome status not only regulates aneuploidy development but also controls faithful chromosomal inheritance. ${ }^{18,20}$ Moreover, some observations have indicated that $\mathrm{CD}$ can also induce an altered immune phenotype.

With regard to aneuploidy, Senovilla et al. first discovered the connection between nonphysiological tetraploidy and immunosurveillance. In immunodeficient mice, elevated exposure of calreticulin (CALR) on the cell membrane endows hyperploid cancer cells with immunogenicity, possibly by constitutively increasing endoplasmic reticulum (ER) stress. CALR facilitates dendritic cell recognition of neoplasm antigens, which ultimately induces an antitumor immune response and results in suppression of tumor growth via cytotoxic CD8 + T cells. In contrast, hyperploid cells can generate tumors only after a delay in immunocompetent mice; the resulting tumors exhibit reduced DNA content, ER stress, and the exposure of CALR. ${ }^{83}$ Hence, aneuploidy enables cells to escape from the immunosurveillance system. Careful analysis of the components involved in the immune microenvironment has revealed that aneuploidy is positively associated with the overall tumor leukocyte fraction and is consistent with the activation of inflammatory signaling pathways. Macrophages are the most important components of the aneuploid tumor microenvironment, which is distinguished by an immunosuppressive phenotype that involves activation of tumor growth factor- $\beta$ (TGF- $\beta$ ). ${ }^{84}$ Moreover, highly aneuploid tumors decrease $\mathrm{CD}^{+} \mathrm{T}$ cell infiltration to reduce the response to immunotherapy. ${ }^{85}$ Likewise, tumors with aneuploidy exhibit suppressive MHC class I antigen presentation and defective immunogenicity to evade antitumor immune responses. ${ }^{86}$ These interesting correlations require to establish animal models that can be used to dissect the reciprocal 


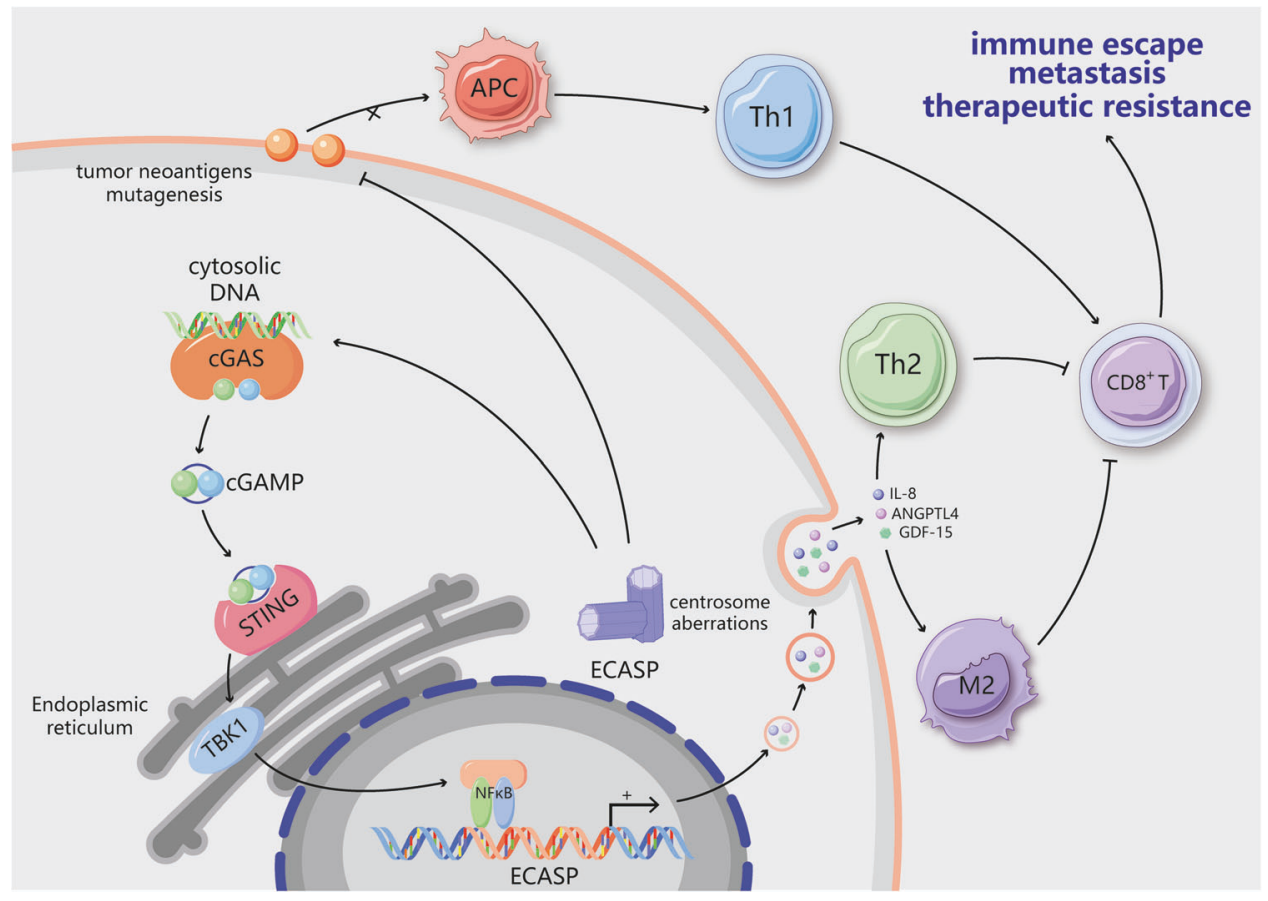

Fig. 3 Centrosome aberrations trigger an immunosuppressive microenvironment. Centrosome aberrations can result in the accumulation of double-stranded DNA (dsDNA) in the cytosol. The presence of cytosolic dsDNA activates the cGAS-STING pathway. In cancer cells with centrosome dysfunction, however, alternative inflammatory STING-dependent signaling, such as NF- $\kappa B$ signaling, is activated. Chronic NF- $\mathrm{BB}$ activation has been shown to mediate the extra centrosome-associated secretory phenotype (ECASP) by affecting proteins including IL-8, GDF-15 and ANGPTL4. IL-8 is also one of the components of the senescence-associated secretory phenotype (SASP); it recruits Th2 cells and M2 macrophages to shape the immunosuppressive microenvironment. Additionally, centrosome aberrations contribute to decreased tumor neoantigen expression and mutagenesis, thereby suppressing MHC class I antigen presentation and decreasing CD8+ T cell infiltration to enable evasion of antitumor immune responses. Ultimately, centrosome abnormalities can lead to immune escape, distant metastasis and therapeutic resistance

crosstalk between aneuploid tumor cells and the corresponding immune microenvironment.

The effects of centrosome amplification on CIN have been elucidated. In tumor tissues, centrosome abnormalities in both size and number are significantly positively correlated with CIN independent of p53 mutation. ${ }^{18,87}$ A study conducted to define the underlying mechanism indicated that extra centrosomes tend to promote chromosome mis-segregation during bipolar cell division. ${ }^{49}$ Subsequently, many research groups have described a direct mechanism through which errors related to mitotic CIN generate DNA breaks via the formation of structures called micronuclei during anaphase arrest. ${ }^{88}$ The envelopes surrounding these micronuclei are prone to rupture, which causes the genomic contents to be exposed to the cytosol. ${ }^{89}$ dsDNA in micronuclei activates the cGAS-STING pathway when dsDNA makes contact with the cytosol during interphase. ${ }^{16}$ cGAS first detects cytosolic dsDNA, causing cGAMP (a cyclic dinucleotide) to be generated, which in turn promotes STING perinuclear localization to the ER membrane ${ }^{90}$ (Fig. 3). STING mediates the transcriptional activation of inflammatory pathways, including the type I interferon signaling pathway, and establishment of the senescenceassociated secretory phenotype (SASP). Through fluorescenceactivated cell sorting (FACS) and subsequent single-cell RNA sequencing of $\mathrm{RNaseH}^{-1-} \mathrm{MEFs}$, Mackenzie et al. elegantly revealed that proinflammatory interferon-stimulated genes (ISGs), including CCL5 and CXCL10, are induced only in cells with micronuclei. ${ }^{91}$ Moreover, chromosome-tracking experiments have shown that the same chromosomes undergoing aberrant separation are eventually fragmented into cytoplasmic chromatin, ${ }^{16}$ indicating a direct connection between chromosome missegregation and innate immune signaling. Taken together, these findings indicate that CIN caused by the centrosome could activate immune signaling pathways via the cGAS-STING pathway.

\section{THE ECASP LINKS SENESCENCE WITH THE IMMUNE MICROENVIRONMENT}

Recently, the extra centrosome-associated secretory pathway (ECASP) has been identified as a distinct secretory phenotype including diverse extracellular matrix (ECM)-associated factors generated by extra centrosomes. ${ }^{28}$ It has also been demonstrated that centrosome amplification facilitates the release of proinvasive factors to trigger non-cell-autonomous invasion ${ }^{28}$ (Fig. 3). Conditioned media derived from cells with multiple centrosomes can promote the generation of invadopodia in normal cells. Furthermore, cells with additional centrosomes have been shown to release a variety of proinvasive factors that are associated with tumorigenesis and with the invasion and migration of cancer cells (e.g., IL-8, ANGPTL4, and GDF-15). The release of proinvasive factors does not require Rac1 signaling; however, Rac1 signaling is required for the formation of invadopodia in response to these secreted factors in cells with normal centrosomes. This finding indicates that the non-cell-autonomous ECASP differs from the previously reported pathway that promotes the cell-autonomous formation of invadopodia in cells with additional centrosomes. ${ }^{60}$ Although how the additional centrosomes promote the ECASP remains unknown, it has been determined that the response depends in part on increases in the levels of ROS in centrosomeamplified cells. ${ }^{28}$ Similarly, aging cells generate a complex mixture of soluble and insoluble factors that comprise the SASP, ${ }^{92}$ including cytokines, chemokines and other signaling molecules. The specific constitution of the SASP varies depending on the type of cell and the agent inducing senescence. IL- 8 is one of the 
Table 1. Potential centrosome-targeted therapy in cancer

\begin{tabular}{|c|c|c|c|}
\hline Agent & Mechanisms & Preclinical and clinical effects & Reference \\
\hline Taxanes paclitaxel & $\begin{array}{l}\text { Inhibits tubulin polymerization and indirectly } \\
\text { induce multipolarity spindles }\end{array}$ & $\begin{array}{l}\text { A chemotherapy medication used to treat a } \\
\text { number of types of cancer }\end{array}$ & $\begin{array}{l}\text { Abal et al. }{ }^{110} \\
\text { Zhu et al. }{ }^{109}\end{array}$ \\
\hline GF-15 & Inhibits centrosomal clustering & Inhibits the proliferation of tumor cells in vitro & $\begin{array}{l}\text { Raab et al. }{ }^{100} \\
\text { Zacharaki } \\
\text { et al. }{ }^{108}\end{array}$ \\
\hline VR23 & $\begin{array}{l}\text { Targets the catalytic } \beta 2 \text { subunit of the } 20 \mathrm{~S} \\
\text { proteasome and induces an aberrant } \\
\text { centrosome amplification cycle }\end{array}$ & $\begin{array}{l}\text { Kills multiple myeloma cells and metastatic } \\
\text { breast cancer cells in vitro and in vivo, and } \\
\text { enhances the antitumor activity of paclitaxel }\end{array}$ & Pundir et al. ${ }^{107}$ \\
\hline CCCI-01 & Blocks centrosome clustering & Recedes the tumor growth in vitro & $\begin{array}{l}\text { Kawamura } \\
\text { et al. }{ }^{106}\end{array}$ \\
\hline CP-673451 crenolanib & Cofilin-mediated cortical actin destabilization & Inhibit the tumor growth in vitro & $\begin{array}{l}\text { Konotop } \\
\text { et al. }{ }^{105}\end{array}$ \\
\hline CFI-400945 & A potent and selective PLK4 inhibitor & $\begin{array}{l}\text { Significantly inhibits tumor growth in vitro } \\
\text { and in vivo }\end{array}$ & Mason et al. ${ }^{104}$ \\
\hline $\begin{array}{l}\text { SK461364A TKM-080301 GW843682 } \\
\text { purpurogallin poloxin }\end{array}$ & PLK 1 inhibitor & $\begin{array}{l}\text { Significantly inhibits multiple tumor growth } \\
\text { in vitro and in vivo are being evaluated in } \\
\text { phase I or II study }\end{array}$ & Liu et al. ${ }^{103}$ \\
\hline Olaparib Phenanthrene AZ0108 & PARP inhibitors block centrosome clustering & $\begin{array}{l}\text { Improve progression-free survival in women } \\
\text { with ovarian cancer; enhances the therapeutic } \\
\text { efficacy of immune checkpoint blockade }\end{array}$ & $\begin{array}{l}\text { Stewart et al. }{ }^{102} \\
\text { Shen et al. }{ }^{101} \\
\text { Castiel et al. }{ }^{98} \\
\text { Johannes } \\
\text { et al. } .^{111}\end{array}$ \\
\hline
\end{tabular}

components of the SASP and can promote invasion of the basement membrane by precancerous epithelial cells. ${ }^{93}$ This suggests that IL-8 may connect the ECASP with the SASP. The mechanism involves activation of CGAS/STING and NF-KB and accumulation of these proteins in chromatin components, which may be the point of convergence for centrosome abnormalities, the immune microenvironment and senescence. Accumulating evidence has indicated that tumors can achieve tolerance via various mechanisms, including through adapting their response to cytosolic DNA signaling or obtaining genomic copy number heterogeneity, to avoid activating harmful components of innate immunity. Although tumor cells may inhibit type I interferon signaling induced by cytosolic DNA, activation of STING facilitates other inflammatory pathways in a tumor cell-autonomous manner. Despite the lack of a significant association between STING and ISGs, there is a significant association between the mRNA levels of cGAS and STING and a positive association between the expression of these genes and the SASP, ${ }^{94}$ which is consistent with the reported effect of the CGAS-STING pathway on cellular senescence. ${ }^{95}$ STING facilitates signaling through many proinflammatory transcription factor-related pathways, such as the canonical and noncanonical NF-kB pathways. ${ }^{96}$ Although the functions of these factors overlap, different outcomes can result from their various effects downstream of STING. For example, the classic NF-KB pathway triggers the generation of SASP inflammatory factors through cytosolic DNA signaling in primary human lung IMR90 fibroblasts. ${ }^{94,95}$ Additionally, through low-level and persistent activation of CGAS-STING, the noncanonical NF-KB pathway is enhanced and has a crucial effect on the migration and invasion ability of MDA-MB-231 cells. ${ }^{16}$ These pathway-specific functions do not seem to be associated with interferon-regulatory factor 3 (IRF3). Although the role of the ECASP in tumor development remains unknown, this pathway participates in a mechanism by which additional centrosomes trigger the paracrine invasion of nearby cells with normal centrosomes. ${ }^{28} \mathrm{~A}$ small fraction of tumor cells undergoing chromosome mis-segregation may be required to generate SASP-associated cytokines that attract immune cells to the tumor microenvironment. The inflammatory microenvironment could in turn result in aneuploidy and propagate CIN in tumor cells via direct genotoxic stress or induction of epithelial-mesenchymal transition (EMT), forming a feed-forward loop. ${ }^{97}$ It would be interesting to explore the consequences of structural centrosome alterations in order to determine whether they are similar to those of numerical alterations that induce a pro-invasive secretory phenotype.

\section{CENTROSOME ABNORMALITIES AND CANCER THERAPY}

The recent development of centrosome clustering inhibitors might enable validation of the role of centrosomes in cancer in vivo. Some compounds that trigger multipolar mitosis preferentially in cancer cells, such as GF-15 (a derivative of griseofulvin), poly(ADP-ribose) polymerase (PARP) inhibitors and paclitaxel (i.e., Taxol), have been developed ${ }^{98-111}$ (Table 1). Cells with additional centrosomes exhibit increased sensitivity to such inhibitors, but it is uncertain whether the amplification of centrosomes in tumor cells causes this sensitivity. Another possibility is that these agents impact microtubule dynamics to lead to the development of multipolar spindles rather than causing the production of extra centrioles. Taxanes, natural antitumor drugs that have been shown to stabilize microtubules, obstruct cell cycle progression by inducing centrosome-related abnormalities, specifically aberrant spindles, and by inhibiting spindle microtubule dynamics. ${ }^{10}$ Paclitaxel, a prototypical taxane antitumor drug that inhibits tubulin polymerization, can induce abnormal multipolar spindle formation to sustain cleavage failure and cause gradual cell death. ${ }^{109}$ Griseofulvin has many properties in common with paclitaxel. Griseofulvin facilitates microtubule instability at low concentrations, and the griseofulvin binding site on tubulin is the same as the Taxol binding site, thereby indicating that griseofulvin has similar effects in inducing multipolar spindle formation. ${ }^{108} \mathrm{GF}-15$, a derivative of griseofulvin, potently inhibits centrosomal clustering, thereby suppressing tumor cell growth in vitro and in vivo. ${ }^{100}$ In addition, a quinoline-sulfonyl hybrid proteasome inhibitor, VR23, targets the catalytic $\beta 2$ subunit of the $20 \mathrm{~S}$ proteasome and induces an aberrant centrosome amplification cycle by promoting the accumulation of ubiquitinated cyclin E to selectively kill cancer cells. ${ }^{107}$ Furthermore, centrosome clustering chemical inhibitor-01 (CCCl-01) induces multipolar spindle formation and inhibits clonogenic growth of BT-549 
breast cancer cells, which have extra centrosomes but retain bipolar spindles similar to those in normal epithelial cells. ${ }^{106}$ Likewise, CP-673451 and crenolanib have been discovered to be robust inhibitors of centrosome clustering with selective cytotoxicity toward cells with extra centrosomes. Mechanistically, both compounds induce mitotic spindle multipolarity via cofilinmediated cortical actin destabilization. ${ }^{105}$ Given the key roles of PLKs in centrosome maturation, some targeted PLK1 and PLK4 inhibitors have been developed to significantly inhibit tumor growth and are currently in the clinical development stage. ${ }^{103,104}$ Ultimately, PARP-1 localizes to centrosomes and catalyzes poly (ADP-ribosyl)ation of centrosomal p53, which is involved in regulation of centrosome duplication and monitoring of chromosomal stability. ${ }^{112}$ Moreover, PARP inhibitors have been discovered to inhibit centrosome clustering and exclusively eradicate multicentrosomal human cancer cells. ${ }^{98,111}$ Hence, PARP inhibitor treatment could be a new, selective and efficient centrosometargeting therapy for a wide range of human cancers.

Aside from acting through these well-established mechanisms of cellular action, inhibitors can affect chromosome segregation by enhancing CGAS-STING pathway signaling, resulting in antitumor immunity. Zierhut et al. showed that STING is an essential determining factor of mitotic cell death in Taxol-treated breast cancer cells in vitro. ${ }^{113}$ Similarly, recent studies have revealed that PARP inhibitors can affect cGAS-STING signaling and antitumor immunity, as indicated by assessments of the tumor response in mouse models of transplantable ovarian and colorectal cancers. ${ }^{101,102}$ Regardless of the associated challenges, it is obvious that identification of agents that rapidly and precisely target centrosomes will be clinically beneficial for certain patients. Additionally, paclitaxel and PARP inhibitors have been shown to enhance immune checkpoint blockade in multiple cancers. ${ }^{114,115}$ Hence, targeted centrosome treatments can promote antitumor responses, and combination therapies are a promising new avenue.

\section{CONCLUSION AND PERSPECTIVE}

The effect of centrosomes on senescence is an important research topic that has been widely ignored. Although further studies are needed, the existing evidence suggests that $C D$ is associated with cellular senescence. We suggest that $C D$ is another form of stress, in addition to well-known cellular stresses such as DNA damage, oxidative stress, oncogenic activation and tumor suppressor overexpression, that can prevent cell cycle arrest and cellular senescence. Notably, increasing amounts of evidence have indicated that p53 is a common protein among these diverse pathways. In response to different sources of stress, p53 accumulates rapidly in the centrosome. Subsequently, phosphorylation of centrosomal $\mathrm{p} 53$ by different kinases at specific residues mediates downstream events, including p21 and Rb stimulation, to cause permanent cell cycle arrest and cellular senescence. Although the regulatory role of nuclear p53 in the transcription process is well understood, the biological function of this protein in the centrosome is worth further study. For example, the mechanisms by which p53 shuttles into and out of the centrosome and the specific effect of p53 on senescence deserve further investigation. In addition, whether activation of the autophagic response can delay CD-mediated senescence warrants investigation. Exploration of these topics will likely provide useful insights into the relationships among cellular senescence, cell death, and uncontrolled cell growth, which are all closely associated with proper centrosome function.

Understanding the dual role of $C D$ as both an innate immune signaling activator and a tumor adaptation mediator is crucial for the development of appropriate therapeutic targets. Although recent findings have improved our understanding of the mechanisms underlying $C D$ in biological systems, the clinical application of CD-targeted therapies is only in the beginning stages. Further advances in $C D$ research are essential for enhancing our ability to prevent tumor cells from adapting to cytosolic DNA and to provide therapeutic benefits by targeting the lethal features of cancer. Furthermore, successful treatment hinges on the development of drugs that can activate autophagy to remove abnormal centrosomes and promote antitumor immune responses. Given the widespread distribution of $C D$ in human cancers, CD-targeted therapies likely have the capacity to improve clinical outcomes, such as by minimizing therapeutic resistance, ameliorating advanced and metastatic disease and enhancing systemic antitumor immunity.

\section{ACKNOWLEDGEMENTS}

We thank the professional English editing service American Journal Experts for assistance in improving the language quality. This work was partially supported by National Natural Science Foundation of China (NSFC) Grant No. 81471781 and National Major Scientific Instruments and Equipment Development Projects Grant No. 2012 YQ160203 to Dr. Shengrong Sun. This work was also supported by National NSFC Grant No. 81903166 and Hubei Province health and family planning scientific research project Grant No. WJ2019Q044 to Dr. Si Sun.

\section{AUTHOR CONTRIBUTIONS}

Q.W. and Sh.S. conceived and performed the review. Q.W. and B.L. wrote the manuscript. L.L. drew all the figures. Si.S. assisted in improving the language quality and provided financial support. All authors read and approved the final manuscript.

\section{ADDITIONAL INFORMATION}

Competing interests: The authors declare no competing interests.

\section{REFERENCES}

1. Doxsey, S., McCollum, D. \& Theurkauf, W. Centrosomes in cellular regulation. Annu. Rev. Cell Dev. Biol. 21, 411-434 (2005).

2. Banterle, N. \& Gonczy, P. Centriole biogenesis: from identifying the characters to understanding the plot. Annu. Rev. Cell Dev. Biol. 33, 23-49 (2017).

3. Zyss, D. \& Gergely, F. Centrosome function in cancer: guilty or innocent? Trends Cell Biol. 19, 334-346 (2009).

4. Weaver, B. A. et al. Aneuploidy acts both oncogenically and as a tumor suppressor. Cancer Cell. 11, 25-36 (2007).

5. Sotillo, R. et al. Mad2 overexpression promotes aneuploidy and tumorigenesis in mice. Cancer Cell. 11, 9-23 (2007).

6. Thompson, S. L. \& Compton, D. A. Examining the link between chromosomal instability and aneuploidy in human cells. J. Cell Biol. 180, 665-672 (2008).

7. Bakhoum, S. F. \& Cantley, L. C. The multifaceted role of chromosomal instability in cancer and its microenvironment. Cell 174, 1347-1360 (2018).

8. Sieben, C. J., Sturmlechner, I., van de Sluis, B. \& van Deursen, J. M. Two-step senescence-focused cancer therapies. Trends Cell Biol. 28, 723-737 (2018).

9. Ohshima, S. \& Seyama, A. Cellular aging and centrosome aberrations. Ann. N. Y. Acad. Sci. 1197, 108-117 (2010).

10. Hossain, D. \& Tsang, W. Centrosome dysfunction and senescence: coincidence or causality. Aging Sci. 1, 2 (2013).

11. Scheer, U. Historical roots of centrosome research: discovery of Boveri's microscope slides in Wurzburg. Philos. Trans. R. Soc. Lond. B Biol. Sci. 369, 20130469 (2014).

12. Levine, M. S. et al. Centrosome amplification is sufficient to promote spontaneous tumorigenesis in mammals. Dev. Cell 40, 313-322.e315 (2017).

13. Sercin, O. et al. Transient PLK4 overexpression accelerates tumorigenesis in p53deficient epidermis. Nat. Cell Biol. 18, 100-110 (2016).

14. Castellanos, E., Dominguez, P. \& Gonzalez, C. Centrosome dysfunction in Drosophila neural stem cells causes tumors that are not due to genome instability. Curr. Biol. 18, 1209-1214 (2008).

15. Jusino, S., Fernandez-Padin, F. M. \& Saavedra, H. I. Centrosome aberrations and chromosome instability contribute to tumorigenesis and intra-tumor heterogeneity. J. Cancer Metastasis Treat. 4, 43 (2018).

16. Bakhoum, S. F. et al. Chromosomal instability drives metastasis through a cytosolic DNA response. Nature 553, 467-472 (2018).

17. Coquel, F. et al. SAMHD1 acts at stalled replication forks to prevent interferon induction. Nature 557, 57-61 (2018).

18. Lingle, W. L. et al. Centrosome amplification drives chromosomal instability in breast tumor development. Proc. Natl. Acad. Sci. USA 99, 1978-1983 (2002). 
19. Kerketta, L. S. et al. Centrosome aberration frequency and disease association in B-acute lymphoblastic leukemia. In Vivo 31, 215-220 (2017).

20. Yamamoto, Y. et al. Centrosome hyperamplification predicts progression and tumor recurrence in bladder cancer. Clin. Cancer Res. 10, 6449-6455 (2004).

21. Jung, C. K. et al. Centrosome abnormalities in non-small cell lung cancer: correlations with DNA aneuploidy and expression of cell cycle regulatory proteins. Pathol. Res. Pract. 203, 839-847 (2007).

22. Kramer, A. et al. Centrosome aberrations as a possible mechanism for chromosomal instability in non-Hodgkin's lymphoma. Leukemia 17, 2207-2213 (2003).

23. Chng, W. J. et al. The centrosome index is a powerful prognostic marker in myeloma and identifies a cohort of patients that might benefit from aurora kinase inhibition. Blood 111, 1603-1609 (2008).

24. Neben, K. et al. Centrosome aberrations in acute myeloid leukemia are correlated with cytogenetic risk profile. Blood 101, 289-291 (2003).

25. Schnerch, D. \& Nigg, E. A. Structural centrosome aberrations favor proliferation by abrogating microtubule-dependent tissue integrity of breast epithelial mammospheres. Oncogene 35, 2711-2722 (2016).

26. Marteil, G. et al. Over-elongation of centrioles in cancer promotes centriole amplification and chromosome missegregation. Nat. Commun. 9, 1258 (2018).

27. Ganier, O. et al. Structural centrosome aberrations promote non-cellautonomous invasiveness. EMBO J. 37, e98576 (2018).

28. Arnandis, T. et al. Oxidative stress in cells with extra centrosomes drives noncell-autonomous invasion. Dev. Cell 47, 409-424 e409 (2018).

29. Godinho, S. A., Kwon, M. \& Pellman, D. Centrosomes and cancer: how cancer cells divide with too many centrosomes. Cancer Metastasis Rev. 28, 85-98 (2009).

30. Fukasawa, K. Oncogenes and tumour suppressors take on centrosomes. Nat. Rev. Cancer 7, 911-924 (2007).

31. Denu, R. A. et al. Centriole overduplication is the predominant mechanism leading to centrosome amplification in melanoma. Mol. Cancer Res. 16, 517-527 (2018).

32. Brownlee, C. W. \& Rogers, G. C. Show me your license, please: deregulation of centriole duplication mechanisms that promote amplification. Cell Mol. Life Sci. 70, 1021-1034 (2013).

33. Nigg, E. A. \& Stearns, T. The centrosome cycle: centriole biogenesis, duplication and inherent asymmetries. Nat. Cell Biol. 13, 1154-1160 (2011).

34. Holland, A. J., Lan, W. \& Cleveland, D. W. Centriole duplication: a lesson in selfcontrol. Cell Cycle 9, 2731-2736 (2010).

35. Habedanck, R., Stierhof, Y. D., Wilkinson, C. J. \& Nigg, E. A. The Polo kinase Plk4 functions in centriole duplication. Nat. Cell Biol. 7, 1140-1146 (2005).

36. O'Connell, K. F. et al. The C. elegans zyg-1 gene encodes a regulator of centrosome duplication with distinct maternal and paternal roles in the embryo. Cell 105, 547-558 (2001)

37. Holland, A. J. et al. Polo-like kinase 4 kinase activity limits centrosome overduplication by autoregulating its own stability. J. Cell Biol. 188, 191-198 (2010)

38. Brownlee, C. W., Klebba, J. E., Buster, D. W. \& Rogers, G. C. The protein phosphatase $2 \mathrm{~A}$ regulatory subunit twins stabilizes Plk4 to induce centriole amplification. J. Cell Biol. 195, 231-243 (2011).

39. Guderian, G., Westendorf, J., Uldschmid, A. \& Nigg, E. A. Plk4 transautophosphorylation regulates centriole number by controlling betaTrCPmediated degradation. J. Cell Sci. 123, 2163-2169 (2010).

40. Li, J. et al. SAK, a new polo-like kinase, is transcriptionally repressed by $\mathrm{p} 53$ and induces apoptosis upon RNAi silencing. Neoplasia 7, 312-323 (2005).

41. Fukasawa, K. et al. Abnormal centrosome amplification in the absence of p53. Science 271, 1744-1747 (1996).

42. Marthiens, V. et al. Centrosome amplification causes microcephaly. Nat. Cell Biol. 15, 731-740 (2013)

43. Borel, F., Lohez, O. D., Lacroix, F. B. \& Margolis, R. L. Multiple centrosomes arise from tetraploidy checkpoint failure and mitotic centrosome clusters in p53 and RB pocket protein-compromised cells. Proc. Natl. Acad. Sci. USA 99, 9819-9824 (2002).

44. Ganem, N. J., Storchova, Z. \& Pellman, D. Tetraploidy, aneuploidy and cancer. Curr. Opin. Genet. Dev. 17, 157-162 (2007).

45. Davoli, T. \& de Lange, T. Telomere-driven tetraploidization occurs in human cells undergoing crisis and promotes transformation of mouse cells. Cancer Cell. 21, 765-776 (2012).

46. Fujiwara, T. et al. Cytokinesis failure generating tetraploids promotes tumorigenesis in p53-null cells. Nature 437, 1043-1047 (2005)

47. Krzywicka-Racka, A. \& Sluder, G. Repeated cleavage failure does not establish centrosome amplification in untransformed human cells. J. Cell Biol. 194, 199-207 (2011).

48. Vitale, I. et al. Multipolar mitosis of tetraploid cells: inhibition by p53 and dependency on Mos. EMBO J. 29, 1272-1284 (2010).
49. Ganem, N. J., Godinho, S. A. \& Pellman, D. A mechanism linking extra centrosomes to chromosomal instability. Nature 460, 278-282 (2009).

50. Brouwer, M. et al. Serum-dependent "cannibalism" and autodestruction in cultures of human small cell carcinoma of the lung. Cancer Res. 44, 2947-2951 (1984).

51. Overholtzer, M. et al. A nonapoptotic cell death process, entosis, that occurs by cell-in-cell invasion. Cell 131, 966-979 (2007).

52. Hamann, J. C. et al. Entosis is induced by glucose starvation. Cell Rep. 20, 201-210 (2017).

53. Durgan, J. et al. Mitosis can drive cell cannibalism through entosis. Elife 6, e27134 (2017).

54. Krajcovic, M. et al. A non-genetic route to aneuploidy in human cancers. Nat. Cell Biol. 13, 324-330 (2011).

55. Florey, O. et al. Autophagy machinery mediates macroendocytic processing and entotic cell death by targeting single membranes. Nat. Cell Biol. 13, 1335-1343 (2011).

56. Tang, C. J. et al. CPAP is a cell-cycle regulated protein that controls centriole length. Nat. Cell Biol. 11, 825-831 (2009).

57. Kohlmaier, G. et al. Overly long centrioles and defective cell division upon excess of the SAS-4-related protein CPAP. Curr. Biol. 19, 1012-1018 (2009).

58. D'Assoro, A. B. et al. Amplified centrosomes in breast cancer: a potential indicator of tumor aggressiveness. Breast Cancer Res. Treat. 75, 25-34 (2002).

59. Nigg, E. A. Origins and consequences of centrosome aberrations in human cancers. Int J. Cancer 119, 2717-2723 (2006).

60. Godinho, S. A. et al. Oncogene-like induction of cellular invasion from centrosome amplification. Nature 510, 167-171 (2014).

61. Satyanarayana, A. et al. Telomere shortening impairs organ regeneration by inhibiting cell cycle re-entry of a subpopulation of cells. EMBO J. 22, 4003-4013 (2003).

62. Schmitt, C. A. et al. A senescence program controlled by $\mathrm{p} 53$ and p16INK4a contributes to the outcome of cancer therapy. Cell 109, 335-346 (2002).

63. Pazolli, E. \& Stewart, S. A. Senescence: the good the bad and the dysfunctional. Curr. Opin. Genet. Dev. 18, 42-47 (2008).

64. Estrada, J. C et al. Human mesenchymal stem cell-replicative senescence and oxidative stress are closely linked to aneuploidy. Cell Death Dis. 4, e691 (2013).

65. Parrinello, S. et al. Oxygen sensitivity severely limits the replicative lifespan of murine fibroblasts. Nat. Cell Biol. 5, 741-747 (2003).

66. Robinson, A. R. et al. Spontaneous DNA damage to the nuclear genome promotes senescence, redox imbalance and aging. Redox Biol. 17, 259-273 (2018).

67. Calado, R. T. \& Dumitriu, B. Telomere dynamics in mice and humans. Semin. Hematol. 50, 165-174 (2013).

68. Ong, A. L. C. \& Ramasamy, T. S. Role of Sirtuin1-p53 regulatory axis in aging, cancer and cellular reprogramming. Ageing Res. Rev. 43, 64-80 (2018).

69. Bartkova, J. et al. Oncogene-induced senescence is part of the tumorigenesis barrier imposed by DNA damage checkpoints. Nature 444, 633-637 (2006).

70. Miao, Y. L. et al. Centrosome abnormalities during porcine oocyte aging. Environ. Mol. Mutagen. 50, 666-671 (2009).

71. Eichenlaub-Ritter, U., Stahl, A. \& Luciani, J. M. The microtubular cytoskeleton and chromosomes of unfertilized human oocytes aged in vitro. Hum. Genet. 80, 259-264 (1988).

72. Miao, Y. L., Kikuchi, K., Sun, Q. Y. \& Schatten, H. Oocyte aging: cellular and molecular changes, developmental potential and reversal possibility. Hum. Reprod. Update 15, 573-585 (2009).

73. Schatten, H., Chakrabarti, A. \& Hedrick, J. Centrosome and microtubule instability in aging Drosophila cells. J. Cell Biochem. 74, 229-241 (1999).

74. Manning, J. A. \& Kumar, S. A potential role for NEDD1 and the centrosome in senescence of mouse embryonic fibroblasts. Cell Death Dis. 1, e35 (2010).

75. Srsen, V., Gnadt, N., Dammermann, A. \& Merdes, A. Inhibition of centrosome protein assembly leads to p53-dependent exit from the cell cycle. J. Cell Biol. 174, 625-630 (2006)

76. Mikule, K. et al. Loss of centrosome integrity induces p38-p53-p21-dependent G1-S arrest. Nat. Cell Biol. 9, 160-170 (2007).

77. Schmidt, S. et al. The centrosomal protein TACC3 controls paclitaxel sensitivity by modulating a premature senescence program. Oncogene 29, 6184-6192 (2010).

78. Katayama, H. et al. Phosphorylation by aurora kinase A induces Mdm2-mediated destabilization and inhibition of p53. Nat. Genet. 36, 55-62 (2004).

79. Ohshima, S. Centrosome aberrations associated with cellular senescence and p53 localization at supernumerary centrosomes. Oxid. Med. Cell Longev. 2012, 217594 (2012)

80. Oricchio, E. et al. ATM is activated by default in mitosis, localizes at centrosomes and monitors mitotic spindle integrity. Cell Cycle 5, 88-92 (2006). 
81. Shinmura, K., Bennett, R. A., Tarapore, P. \& Fukasawa, K. Direct evidence for the role of centrosomally localized p53 in the regulation of centrosome duplication. Oncogene 26, 2939-2944 (2007).

82. Gajewski, T. F., Schreiber, H. \& Fu, Y. X. Innate and adaptive immune cells in the tumor microenvironment. Nat. Immunol. 14, 1014-1022 (2013).

83. Senovilla, L. et al. An immunosurveillance mechanism controls cancer cell ploidy. Science 337, 1678-1684 (2012).

84. Thorsson, V. et al. The immune landscape of cancer. Immunity 48, 812-830 e814 (2018).

85. Davoli, T., Uno, H., Wooten, E. C. \& Elledge, S. J. Tumor aneuploidy correlates with markers of immune evasion and with reduced response to immunotherapy. Science 355, eaaf8399 (2017).

86. Tripathi, R. et al. Suppression of tumor antigen presentation during aneuploid tumor evolution contributes to immune evasion. Oncoimmunology 8, 1657374, (2019).

87. Denu, R. A. et al. Centrosome amplification induces high grade features and is prognostic of worse outcomes in breast cancer. BMC Cancer 16, 47 (2016).

88. Crasta, K. et al. DNA breaks and chromosome pulverization from errors in mitosis. Nature 482, 53-58 (2012).

89. Hatch, E. M., Fischer, A. H., Deerinck, T. J. \& Hetzer, M. W. Catastrophic nuclear envelope collapse in cancer cell micronuclei. Cell 154, 47-60 (2013).

90. Cai, X., Chiu, Y. H. \& Chen, Z. J. The cGAS-cGAMP-STING pathway of cytosolic DNA sensing and signaling. Mol. Cell. 54, 289-296 (2014).

91. Mackenzie, K. J. et al. cGAS surveillance of micronuclei links genome instability to innate immunity. Nature 548, 461-465 (2017).

92. Kuilman, T. \& Peeper, D. S. Senescence-messaging secretome: SMS-ing cellular stress. Nat. Rev. Cancer 9, 81-94 (2009).

93. Coppe, J. P. et al. Senescence-associated secretory phenotypes reveal cellnonautonomous functions of oncogenic RAS and the p53 tumor suppressor. PLoS Biol. 6, 2853-2868 (2008).

94. Dou, Z. et al. Cytoplasmic chromatin triggers inflammation in senescence and cancer. Nature 550, 402-406 (2017).

95. Gluck, S. et al. Innate immune sensing of cytosolic chromatin fragments through cGAS promotes senescence. Nat. Cell Biol. 19, 1061-1070 (2017)

96. Abe, T. \& Barber, G. N. Cytosolic-DNA-mediated, STING-dependent proinflammatory gene induction necessitates canonical NF-kappaB activation through TBK1. J. Virol. 88, 5328-5341 (2014).

97. Comaills, V. et al. Genomic instability is induced by persistent proliferation of cells undergoing epithelial-to-mesenchymal transition. Cell Rep. 17, 2632-2647 (2016).

98. Castiel, A. et al. A phenanthrene derived PARP inhibitor is an extra-centrosomes de-clustering agent exclusively eradicating human cancer cells. BMC Cancer 11, 412 (2011).

99. Chen, J. G. \& Horwitz, S. B. Differential mitotic responses to microtubulestabilizing and -destabilizing drugs. Cancer Res. 62, 1935-1938 (2002).

100. Raab, M. S. et al. GF-15, a novel inhibitor of centrosomal clustering, suppresses tumor cell growth in vitro and in vivo. Cancer Res. 72, 5374-5385 (2012).

101. Shen, J. et al. PARPi triggers the STING-dependent immune response and enhances the therapeutic efficacy of immune checkpoint blockade independent of BRCAness. Cancer Res. 79, 311-319 (2019).

102. Stewart, R. A., Pilie, P. G. \& Yap, T. A. Development of PARP and immunecheckpoint inhibitor combinations. Cancer Res. 78, 6717-6725 (2018).
103. Liu, X. Targeting polo-like kinases: a promising therapeutic approach for cancer treatment. Transl. Oncol. 8, 185-195 (2015).

104. Mason, J. M. et al. Functional characterization of CFI-400945, a Polo-like kinase 4 inhibitor, as a potential anticancer agent. Cancer Cell. 26, 163-176 (2014).

105. Konotop, G. et al. Pharmacological inhibition of centrosome clustering by slingshot-mediated cofilin activation and actin cortex destabilization. Cancer Res. 76, 6690-6700 (2016).

106. Kawamura, E. et al. Identification of novel small molecule inhibitors of centrosome clustering in cancer cells. Oncotarget 4, 1763-1776 (2013).

107. Pundir, S. et al. VR23: a quinoline-sulfonyl hybrid proteasome inhibitor that selectively kills cancer via cyclin E-mediated centrosome amplification. Cancer Res. 75, 4164-4175 (2015).

108. Zacharaki, P., Stephanou, G. \& Demopoulos, N. A. Comparison of the aneugenic properties of nocodazole, paclitaxel and griseofulvin in vitro. Centrosome defects and alterations in protein expression profiles. J. Appl Toxicol. 33, 869-879 (2013).

109. Zhu, J. et al. Centrosome impairments and consequent cytokinesis defects are possible mechanisms of taxane drugs. Anticancer Res. 25, 1919-1925 (2005).

110. Abal, M., Andreu, J. M. \& Barasoain, I. Taxanes: microtubule and centrosome targets, and cell cycle dependent mechanisms of action. Curr. Cancer Drug Targets 3, 193-203 (2003).

111. Johannes, J. W. et al. Discovery of AZ0108, an orally bioavailable phthalazinone PARP inhibitor that blocks centrosome clustering. Bioorg. Med. Chem. Lett.25, 5743-5747 (2015)

112. Kanai, M. et al. Involvement of poly(ADP-Ribose) polymerase 1 and poly(ADPRibosyl)ation in regulation of centrosome function. Mol. Cell Biol. 23, 2451-2462 (2003).

113. Zierhut, $C$. et al. The cytoplasmic DNA sensor cGAS promotes mitotic cell death Cell 178, 302-315.e323 (2019).

114. Schmid, P. et al. Atezolizumab and nab-paclitaxel in advanced triple-negative breast cancer. N. Engl. J. Med. 379, 2108-2121 (2018).

115. Karzai, F. et al. Activity of durvalumab plus olaparib in metastatic castrationresistant prostate cancer in men with and without DNA damage repair mutations. J. Immunother. Cancer 6, 141 (2018).

Open Access This article is licensed under a Creative Commons Attribution 4.0 International License, which permits use, sharing, adaptation, distribution and reproduction in any medium or format, as long as you give appropriate credit to the original author(s) and the source, provide a link to the Creative Commons license, and indicate if changes were made. The images or other third party material in this article are included in the article's Creative Commons license, unless indicated otherwise in a credit line to the material. If material is not included in the article's Creative Commons license and your intended use is not permitted by statutory regulation or exceeds the permitted use, you will need to obtain permission directly from the copyright holder. To view a copy of this license, visit http://creativecommons. org/licenses/by/4.0/.

(c) The Author(s) 2020 